\title{
Arthropod Diversity in Horticultural and Silvicultural Crops of Yercaud Hills
}

\author{
M. P. Sugumaran*, A. S. Goveanthan and Ganesh Kumar Gudimetha \\ Department of Environmental Sciences, Tamil Nadu Agricultural University, \\ Coimbatore, India \\ *Corresponding author
}

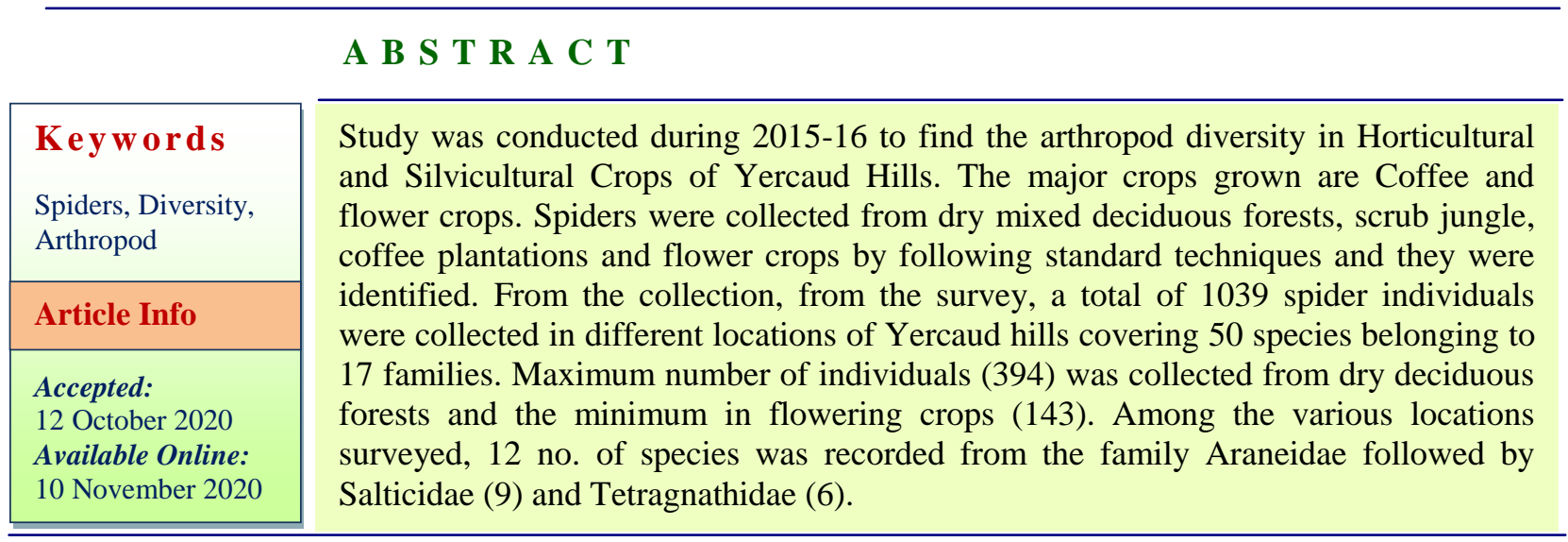

\section{Introduction}

India is exceptionally rich in biodiversity with a very large species composition of 45,000 plant species and 81,000 animal species representing seven per cent of world's flora and 6.5 per cent of world's fauna, and is one of the 12 centres of mega diversity in the world. The natural forest litter ecosystem with its floral diversity and specialized micro niches supports a variety of macro and micro arthropods involving different trophic levels. Arthropod inventories can be good indicators of habitat biodiversity because arthropods respond quickly to environmental changes, since they are highly diverse in nature. Many amphibians, replites, aves, mammals and spiders are good indicators of environmental health. They play important roles in the dynamics of a specific habitat and are sensitive to habitat loss, climatic change and environmental upheavels. This study focuses on the influence of weather parameters on the changes in popupulation and diversity of arthropods in bamboo plantations.

\section{Materials and Methods}

\section{Study area}

\section{Survey of Spiders}

The survey for collection and population assessment of spiders was carried in three 
different locations in dry deciduous forests, coffee plantations and scrub jungles in Yercaud hills in three altitudes i.e. at i) $1000 \mathrm{~m}$ above MSL, ii) $1300 \mathrm{~m}$ above MSL and iii)1500 $\mathrm{m}$ above MSL.

\section{Spider survey}

The study was carried out during 2015-16 in all the crops in Yercaud hills and the specimens were collected by following standard techniques (Sutherland 1996). Individual spider species were collected, identified and biodiversity of spiders was calculated using Shannon wiener index.

The five standard sampling methods such as, Visual searching cum Hand picking, Net sweeping, Beating tray, Pitfall traps and Leaf litter traps. Visual searching cum hand picking and Net sweeping methods were employed for spider collection in this study. Collected arthropods were sorted out based on taxon. Soft bodied insects and spider species were preserved in $70 \%$ ethyl alcohol in glass vials. Insects were identified by following Lefroy (1984), Comstock (1984), Reichard and Davis (1993) and Ayyar (1984) and with the help of spider taxonomists.

\section{Shannon-Wiener Index of Diversity}

Shannon-Wiener Index of Diversity is used to examine the overall community characteristics comparing two or more distinct habitats. It is a measure of the possibility that the next individual will be the same spider species as the previous sample. ShannonWiener Index of diversity was calculated (Batten 1976).

$$
\mathrm{H}^{\prime}=-5 \cdot \text { pi. lnpi }
$$

Where, pi is the proportional abundance of the $i^{\text {th }}$ species $=(n i / N)$.

\section{Results and Discussion}

\section{Spider diversity}

A total of 1039 spider individuals were collected in different locations of Yercaud hills covering 50 species belonging to 17 families (Table 1). Maximum number of individuals (394) was collected from dry deciduous forests and the minimum in flowering crops (143). Among the various locations surveyed, 12 no. of species was recorded from the family Araneidae followed by Salticidae (9) and Tetragnathidae (6). Spider diversity was maximum in Dry mixed deciduous forests in Kotachedu as per Shannon's index (1.014) (Table 1) and minimum in flowering crops (0.740). Among the crops, Coffee plantations has maximum spider diversity (1.184) followed by Fruit trees (1.184) and flowering crops has minimum spider diversity (0.874). Shunmugavelu et al., (2011) reported 20 species of spiders in bhendi field of Chekkanoorani near Madurai representing eight families and 15 genera. The most frequently encountered species are Argiope pulchella (Thorell), Argiope lobata (Pallas), Cyrtophora citricola (Forskal), Leucauge decorata (Blackwall), Neoscona crucifera (Lucas), Gasteracantha aciculata (Pocock), Cyclosa conica (Pallas), Hersilia savignyi (Lucas), Latrodectus hasselti (Thorell), Nephila pilipes (Fabricius), Peucetia viridana (Stoliczka), Peucetia graminea (Pocock), Oxyopes ratnae (Tikader), Stegodyphus sarasinorum (Karsch), Heteropoda phasma (Simon), Heteropoda venatoria (Linnaeus), Micrommata virescens (Clerck), Tetragnatha mandibulata (Walckenaer), Tetragnatha anguilla (Thorell) and Leucauge festiva (Blackwall). Ganesan and Shunmugavelu (2012) recorded a faunal survey of 25 species of spiders belonging to 21 genera and 10 families in Perumalmalai forest area, Tamil Nadu. 
Table.1 Total number of species and specimens collected from different locations

\begin{tabular}{|c|c|c|c|c|c|c|c|c|c|c|c|c|c|c|c|c|c|c|c|c|c|c|c|c|}
\hline \multirow[t]{3}{*}{ Spider family } & \multicolumn{8}{|c|}{ Yercaud } & \multicolumn{8}{|c|}{ Muluvi } & \multicolumn{8}{|c|}{ Kotachedu } \\
\hline & \multicolumn{2}{|c|}{ DMDF } & \multicolumn{2}{|c|}{$\mathbf{C P}$} & \multicolumn{2}{|c|}{ FC } & \multicolumn{2}{|c|}{ SJ } & \multicolumn{2}{|c|}{ DMDF } & \multicolumn{2}{|c|}{$\mathrm{CP}$} & \multicolumn{2}{|c|}{ FC } & \multicolumn{2}{|c|}{ SJ } & \multicolumn{2}{|c|}{ DMDF } & \multicolumn{2}{|c|}{$\mathbf{C P}$} & \multicolumn{2}{|c|}{ FC } & \multicolumn{2}{|c|}{ SJ } \\
\hline & $\begin{array}{c}\text { TN } \\
\mathrm{S}\end{array}$ & TSP & $\begin{array}{c}\text { TN } \\
\mathrm{S}\end{array}$ & $\begin{array}{c}\text { TS } \\
\mathrm{P}\end{array}$ & $\begin{array}{c}\mathrm{TN} \\
\mathrm{S}\end{array}$ & $\begin{array}{c}\text { TS } \\
\mathrm{P}\end{array}$ & $\begin{array}{c}\mathrm{TN} \\
\mathrm{S}\end{array}$ & TSP & $\begin{array}{c}\mathrm{TN} \\
\mathrm{S}\end{array}$ & $\begin{array}{c}\text { TS } \\
\mathrm{P}\end{array}$ & $\begin{array}{c}\mathrm{TN} \\
\mathrm{S}\end{array}$ & $\begin{array}{c}\text { TS } \\
\mathrm{P}\end{array}$ & $\begin{array}{c}\mathrm{TN} \\
\mathrm{S}\end{array}$ & $\begin{array}{c}\mathrm{TS} \\
\mathrm{P}\end{array}$ & $\begin{array}{c}\mathrm{TN} \\
\mathrm{S}\end{array}$ & TSP & $\begin{array}{c}\mathrm{TN} \\
\mathrm{S}\end{array}$ & $\begin{array}{c}\mathrm{TS} \\
\mathrm{P}\end{array}$ & $\begin{array}{c}\text { TN } \\
\mathrm{S}\end{array}$ & $\begin{array}{c}\mathrm{TS} \\
\mathrm{P}\end{array}$ & $\begin{array}{c}\text { TN } \\
\mathrm{S}\end{array}$ & $\begin{array}{c}\text { TS } \\
\mathrm{P}\end{array}$ & $\begin{array}{c}\mathrm{TN} \\
\mathrm{S}\end{array}$ & $\begin{array}{c}\mathrm{TS} \\
\mathrm{P}\end{array}$ \\
\hline Agelenidae & 1 & 3 & 1 & 2 & 0 & 0 & 1 & 3 & 1 & 4 & 1 & 3 & 0 & 7 & 1 & 7 & 1 & 5 & 1 & 3 & 0 & 0 & 1 & 10 \\
\hline Araneidae & 9 & 16 & 7 & 12 & 4 & 12 & 8 & 15 & 10 & 29 & 8 & 16 & 4 & 15 & 6 & 22 & 12 & 25 & 10 & 26 & 6 & 10 & 8 & 25 \\
\hline Deinopidae & 1 & 2 & 0 & 0 & 0 & 0 & 1 & 1 & 1 & 2 & 1 & 2 & 1 & 1 & 1 & 1 & 1 & 3 & 0 & 0 & 0 & 0 & 1 & 2 \\
\hline Drymusidae & 0 & 0 & 0 & 0 & 0 & 0 & 1 & 3 & 1 & 2 & 1 & 4 & 0 & 0 & 1 & 3 & 1 & 4 & 0 & 0 & 0 & 0 & 1 & 5 \\
\hline Hersilidae & 2 & 3 & 2 & 5 & 0 & 0 & 1 & 1 & 2 & 3 & 2 & 5 & 0 & 0 & 0 & 0 & 2 & 8 & 2 & 5 & 0 & 0 & 1 & 2 \\
\hline Linyphiidae & 1 & 2 & 1 & 1 & 1 & 2 & 1 & 1 & 1 & 2 & 1 & 1 & 1 & 3 & 0 & 0 & 1 & 4 & 1 & 4 & 1 & 3 & 1 & 4 \\
\hline Lycosidae & 1 & 1 & 1 & 1 & 0 & 0 & 1 & 2 & 1 & 4 & 1 & 1 & 0 & 0 & 1 & 2 & 1 & 6 & 1 & 1 & 0 & 0 & 1 & 2 \\
\hline Pholcidae & 0 & 0 & 0 & 0 & 0 & 0 & 0 & 0 & 2 & 5 & 1 & 1 & 0 & 0 & 0 & 0 & 2 & 7 & 0 & 0 & 0 & 0 & 0 & 0 \\
\hline Sparassidae & 1 & 3 & 1 & 2 & 1 & 2 & 2 & 4 & 2 & 4 & 1 & 2 & 1 & 4 & 1 & 5 & 2 & 8 & 2 & 4 & 1 & 4 & 2 & 6 \\
\hline Scytodidae & 1 & 3 & 0 & 0 & 0 & 0 & 1 & 1 & 1 & 5 & 0 & 0 & 0 & 0 & 0 & 0 & 1 & 5 & 0 & 0 & 0 & 0 & 1 & 2 \\
\hline Salticidae & 5 & 21 & 3 & 11 & 3 & 8 & 6 & 12 & 6 & 38 & 5 & 11 & 4 & 12 & 4 & 12 & 9 & 20 & 6 & 32 & 3 & 6 & 6 & 18 \\
\hline Theridiidae & 1 & 3 & 1 & 1 & 0 & 0 & 1 & 2 & 2 & 4 & 0 & 0 & 1 & 3 & 1 & 4 & 2 & 5 & 1 & 4 & 0 & 0 & 1 & 6 \\
\hline Tetragnathidae & 3 & 15 & 2 & 3 & 2 & 6 & 4 & 9 & 4 & 34 & 3 & 5 & 3 & 15 & 3 & 13 & 6 & 26 & 5 & 51 & 2 & 0 & 4 & 15 \\
\hline Thomisidae & 2 & 8 & 2 & 2 & 3 & 4 & 2 & 3 & 3 & 10 & 1 & 1 & 3 & 2 & 1 & 3 & 4 & 12 & 2 & 9 & 3 & 8 & 2 & 6 \\
\hline Uloboridae & 1 & 2 & 0 & 0 & 0 & 0 & 1 & 1 & 1 & 2 & 1 & 2 & 1 & 1 & 1 & 1 & 1 & 2 & 0 & 0 & 0 & 0 & 1 & 2 \\
\hline H' & \multicolumn{2}{|c|}{0.980} & \multicolumn{2}{|c|}{0.941} & \multicolumn{2}{|c|}{0.749} & \multicolumn{2}{|c|}{1.010} & \multicolumn{2}{|c|}{0.976} & \multicolumn{2}{|c|}{0.991} & \multicolumn{2}{|c|}{0.942} & \multicolumn{2}{|c|}{0.911} & \multicolumn{2}{|c|}{1.014} & \multicolumn{2}{|c|}{0.822} & & & 1. & \\
\hline
\end{tabular}

Where, DMDF -Dry mixed deciduous forests, CP -Coffee plantations, FC-Flowering crops and SJ-Scrub jungle, TNS-Total number of species, TSP-Total number of specimens and H' - Shannon diversity index 
Plate.1 Spider species collected from Yercaud hills in different ecosystems

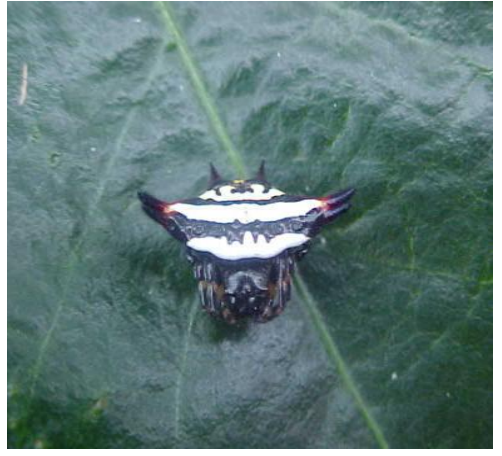

Gasteracantha geminata

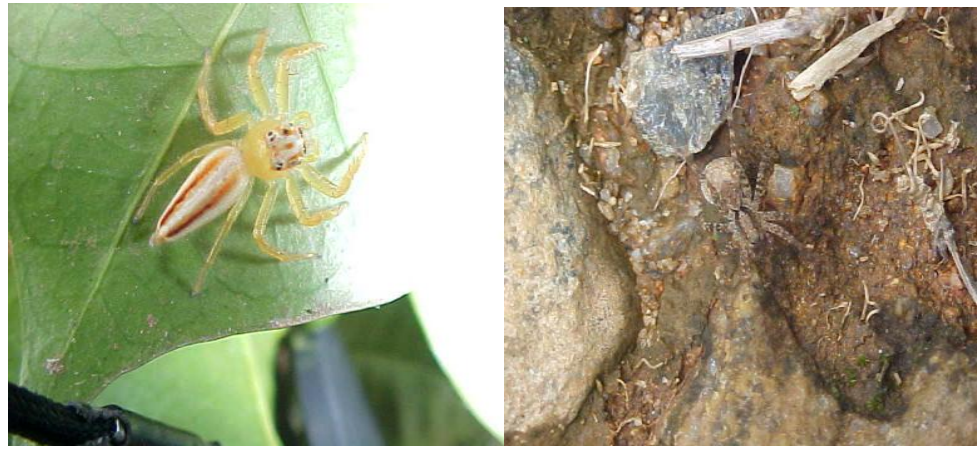

Telamonia dimidiate

Pardosa pseudoannulata

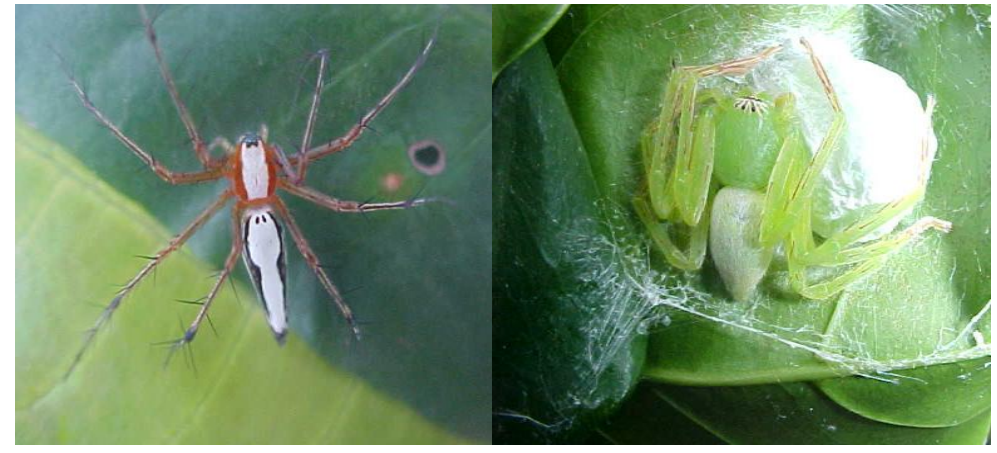

Oxyopes lineatipes
Olios milleti

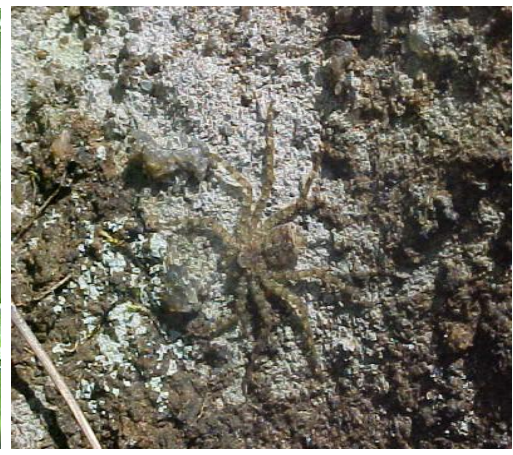

Hersilia savignyi

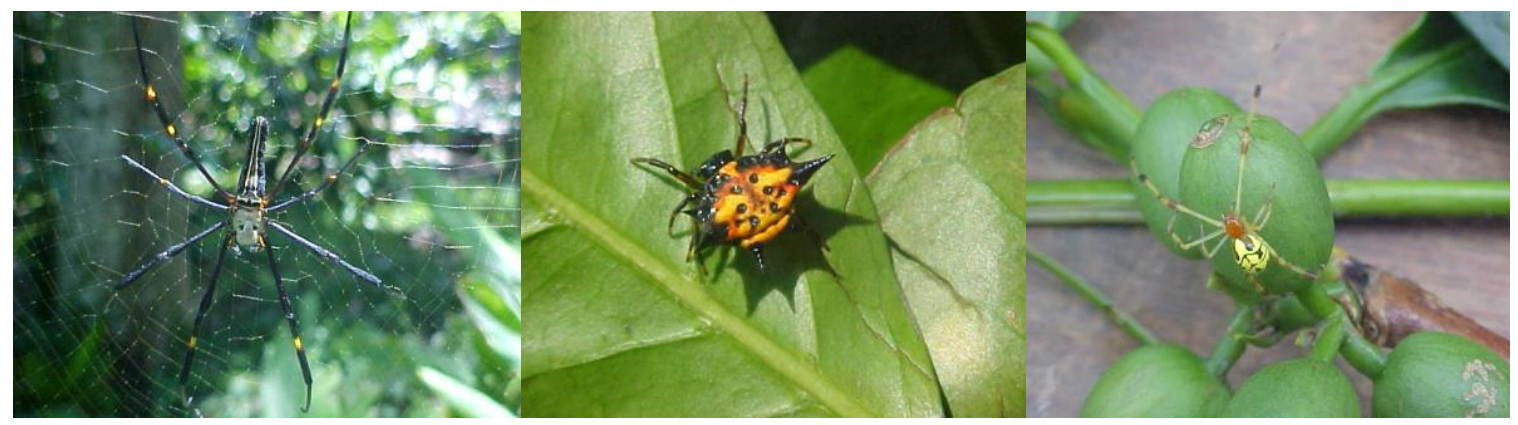

Nephila pilipes

Gasteragantha hasselti

Linyphia urbasae

Climatic factors might influence the abundance and diversity of arthropods by affecting their habitat, movement and altering the suitability of food plants. In another study, it was found that soil arthropod communities were higher in dry season or early rainy season than in middle rainy season (Yang and Tang, 2004) and manmade forest habitat in Menagesha holds the highest diversity of insects in both dry and wet seasons (Tamrat Aydagnhum, 2007).
In conclusion all spiders are predators and feed on a wide prey range consisting of invertebrates, but can also include prey like fishes, birds or even reptiles. Spiders are regarded to be the most abundant and effective predators of insects in many terrestrial ecosystems and have a prey finding ability and capacity to consume greater number of prey than any other predator. They are the only class of arthropods that are entirely predaceous. In this study the forest 
ecosystems recorded maximum spider diversity when compared to the flowering plants.

\section{References}

Ayyar, T.V.R. 1984. Handbook of Economic Entomology for South India, Narendra Publishing House, Delhi, pp.461.

Batten, L.A. 1976. Bird communities of some Killarney woodlands. Proc. Roy. Irish Acad., 76: 285-313.

Comstock, J.H. 1984. An Introduction to Entomology, Satish Book Enterprises, Agra, India, p.840.

Lefroy, H. (1984). Indian Insect Life: Jagmander Book Agency, New Delhi, p.786.

Richards, O.W. and R. J. Davis. (1993). Imms general book of entomology. BI Publications Private Ltd., New Delhi, p.784.
Shunmugavelu, M., Thenmozhi, G. and Karthikeyani, R. 2011. Climatic variation

and diversity of the spider (Araneae) fauna in bhendi fields, Chekkanoorani, Madurai district, Tamilnadu, South India. National Science day and $42^{\text {nd }}$ Aquaterr Annual Conference on Genomic Sciences, M.K University, Madurai, $28^{\text {th }}$ February 2011, abstract, p. 31.

Tamrat Aydagnhum. (2007). Study on Insect diversity of Menagesha forest and Bihere Tsige Public park in wet and dry seasons using Sweep net.Thesis submitted to the Addis Ababa University, pp.1-61.

Yang and Tang (2004). Soil arthropod communities in different successional tropical secondary forests in Xishuangbanna. http://www.ncbi.nlm. nih.gov/pubmed/15362621

\section{How to cite this article:}

Sugumaran, M. P., A. S. Goveanthan and Ganesh Kumar Gudimetha. 2020. Arthropod Diversity in Horticultural and Silvicultural Crops of Yercaud Hills. Int.J.Curr.Microbiol.App.Sci. 9(11): 1724-1728. doi: https://doi.org/10.20546/ijcmas.2020.911.205 